\title{
The Impact of a Digital Vaccine Consent Form in a Large Community Pharmacy Chain
}

Aylin Unal, PharmD*1; Amy Sparkman, PharmD, BCACP², Pramit Nadpara, PhD, MS, BPharm ${ }^{1}$;

Jean-Venable R. Goode, PharmD, BCPS, FAPhA, FCCP

${ }^{1}$ Virginia Commonwealth University; ${ }^{2}$ Kroger Pharmacy, Charlottesville VA

*At the time of writing was a PGY1 Community-Based Resident. Now at Correct Rx Pharmacy Services, Hanover, MD

\begin{abstract}
Background: A large community pharmacy chain implemented a new digital platform to eliminate the need for patients to fill out a traditional vaccine consent form in the pharmacy. The new digital vaccine consent form allowed patients to complete the form online, where it was transmitted directly to the pharmacy's network.

Objectives: To identify the characteristics of patients who used an online digital vaccine consent form to receive vaccinations and to evaluate patient satisfaction and confidence in utilizing the digital vaccine consent form to receive pharmacy services.

Methods: This three-month prospective study was conducted in the Mid-Atlantic division of a large community pharmacy chain. A 16question survey was developed using information from the literature to collect demographic information and patient confidence and satisfaction with the digital vaccine consent form. An email was sent to pharmacy staff containing instructions on the procedure for posting a recruitment flyer, distributing the survey post-vaccination, and how to return completed surveys. Univariate and bi-variate analysis were conducted.
\end{abstract}

Results: Thirty-six participants responded to the survey, majority of participants were female (56\%). Two patients used the digital vaccine consent form; both used because it was more convenient and were likely to use the form again. For those who did not use the digital vaccine consent form, 32\% feel somewhat unconfident in using digital technologies for pharmacy services. A majority of patients prefer to be notified about new online services by email (39\%) or advertisements in the pharmacy (31\%). When asked the likelihood of using the digital vaccine consent form in the future, majority stated unlikely (34\%) or neutral (25\%).

Conclusions: Most participants did not utilize the new digital vaccine form. This provides an opportunity to further engage patients on the availability and use of the digital vaccine consent form in order to advance digital technologies for pharmacy services.

Keywords: digital vaccine consent form, vaccine, confidence, technology

\section{Background}

Vaccinations have become an integral patient care service provided by community-based pharmacists. The main reason that patients choose to receive a vaccine in a community pharmacy is the convenience. ${ }^{1}$ Pharmacists are positioned to serve as educators, facilitators, and administrators to aid in improving vaccine coverage. ${ }^{1,2}$ Although people have positive experiences with pharmacist-provided immunizations, consent forms and wait times still remain a barrier and are considered areas for improvement. ${ }^{1,2}$

A typical vaccine consent form consists of questions and writein responses for the patient to complete and sign prior to receiving a vaccination. Questions on the form include patient demographics and an immunization screening questionnaire. Pharmacist review of the vaccine consent form is integral in ensuring the safety and appropriateness for administering vaccines. Despite the importance of the vaccine consent form, completing paperwork is a main concern for patients and it is recommended for pharmacies to streamline the current documentation process. ${ }^{1}$

Corresponding author: Jean-Venable R. Goode, PharmD, BCPS, FAPhA, FCCP, Professor and Director

PGY-1 Community-Based Pharmacy Residency

Virginia Commonwealth University, Richmond, VA

Email: jrgoode@vcu.edu
A large community pharmacy chain implemented a new digital platform to eliminate the need for patients to fill out a traditional vaccine consent form in the pharmacy. The digital vaccine consent form provides patients the opportunity to complete the consent form online through the website where it is transmitted directly to the pharmacy's network. This will potentially alleviate one barrier to receiving vaccines and allow patients to engage digitally to obtain pharmacy services. ${ }^{1}$ It also provides an opportunity to digitally transform a component of a pharmacy patient care service. ${ }^{3}$

Despite the potential benefits the digital vaccine consent form intends to provide, adoption of a new technology is dependent on a multitude of factors: value, usability, affordability, accessibility, technical support, social support, emotion, independence, experience, and confidence. ${ }^{4}$ By analyzing what makes patients decide to use the digital vaccine consent form, we can determine what can be done to improve patient engagement on digital platforms. Understanding what drives patients to use digital platforms to utilize pharmacy services has important implications for the profession.

\section{Objectives}

The objectives of this study were to identify the characteristics of patients who used an online digital vaccine consent form to receive vaccinations and to evaluate patient satisfaction and confidence in utilizing the digital vaccine consent form to receive pharmacy services. 


\section{Methods}

This three-month prospective survey-based study was conducted from January 20 to April 20, 2020 in the Mid-Atlantic Division of a national large community pharmacy chain. A 16question survey was developed by the investigators using information from the literature. The questions included openended, multiple choice, and write-in responses to collect demographic information and patient confidence and satisfaction with the digital vaccine consent form. Demographic information included age and gender.

An email using store emails was sent to the entire pharmacy staff in the Mid-Atlantic division (110 stores) explaining the purpose of the research project. The email contained instructions on the procedure for posting a recruitment flyer in the private consultation rooms, how to distribute the survey post-vaccination if a patient requested to take the survey, and how to return completed surveys. The pharmacies were not required to participate in the study but became a part of the study only if a patient requested to take the survey.

After receiving a vaccination, patients who requested to take the survey completed it in a private consultation room and placed the survey in an envelope to be collected by pharmacy staff. Once ten surveys were completed, the surveys were mailed to the primary researcher. Survey data was analyzed using Wilcoxon-Mann-Whitney test and descriptive statistics using SPSS version 24. The study was approved by the Virginia Commonwealth University Institutional Review Board.

\section{Results}

Thirty-six participants responded to the survey from multiple pharmacies. The majority of participants were female (56\%) and adults aged 65 years old or above (46\%) (Table 1). Two participants used the digital vaccine consent form; both used the form because it was more convenient and they were likely to use the form again. From the survey, both responded "extremely satisfied" to describe their overall satisfaction with using the form. For those who did not use the digital vaccine consent form, 32\% feel somewhat unconfident in using digital technologies for pharmacy services. A majority of participants prefer to be notified about new online services by email or advertisements in the pharmacy. When asked the likelihood of using the digital vaccine consent form in the future, a majority of responses either said unlikely (34\%) or neutral $(25 \%)$. Comparing male vs. female differences, there was no statistically significant difference in the confidence on using digital technologies $(p<0.590$ ) or likelihood of using the digital vaccine consent form for future vaccinations $(p<0.771)$. Similarly, there was no statistically significant difference in age groups, less than 65 years old vs. 65 years old and older, in confidence on using digital technologies $(p<0.594)$ or likelihood of using the digital vaccine consent form for future vaccinations $(p<0.565)$.

\section{Discussion}

The digital vaccine consent form has the potential to impact pharmacies in alleviating the known barriers of consent forms and wait times when receiving vaccines. ${ }^{1,2}$ Transformation in technology may change the efficiency and accessibility of the pharmacy practice landscape as seen in multiple studies. ${ }^{5,6}$

Davies et al. ${ }^{5}$ explored pharmacists' perception of mobile applications (i.e. 'apps') within the community setting. The study, completed in the United Kingdom (UK), distributed a 30item questionnaire to UK pharmacists. The majority of respondents (78.4\% of 211 participants) were confident in using mobile apps on their technology platform. They also found pharmacists suggested mobile apps will have a greater impact in the future $(p<0.001)$ and as the younger generation ages, mobile apps will be a more accepted method in managing healthcare in the wider population. Although the majority of pharmacists surveyed held a degree of confidence when using mobile apps, $2.5 \%$ of individuals stated they were not at all confident. This further enhances the importance of implementing changes to pharmacy services that can be used by the general population through digital platforms. Even though the digital vaccine consent form was not available through a mobile application it could still be completed through a mobile device such as a cellular telephone.

Velthoven et al. ${ }^{6}$ investigated the question, "how can healthcare organizations compete in a world that is rapidly digitizing?" A workshop was conducted in Switzerland where 12 different stakeholders participated from a wide range of backgrounds such as pharmacy retailers, patients, health insurers, and pharmaceutical companies. Participants worked in groups to assess organizations with digital health products and determine what made them successful. The key success factors were interoperability, low cost, and enough people using the digital product. This work allowed organizations to identify strengths and limitations in their digital initiatives and how to develop new capabilities faster and collaborate with technology organizations. Knowledge about how competitors are advancing digital products as well as the views of the consumer will help with development of digital solutions. With our study, the digital vaccine consent form was newly created so it was essential to get feedback from the users to be able to improve and advance it for the future. However, it was difficult to analyze because not enough people used the digital product to make an assessment.

Our study was limited by the small sample size. The marketing of the new digital vaccine consent form was heaviest prior to influenza season but unfortunately, the Institutional Review Board approval was significantly delayed by the process taking a couple of months for final approval. Therefore, the observational period began later in the influenza vaccination season and fewer people were receiving influenza vaccines during the launch of the study observation period. Collecting data on the total number of vaccinations given during the study 
period would also be useful in determining a response rate and should be considered for future studies. Another limitation was the difficulty with getting engagement from the pharmacy staff to post the recruitment flyer and send completed surveys back to the primary researcher. A suggestion to increase engagement with pharmacy staff would be to reward the pharmacy team with the most collected surveys with a prize, such as a pizza party or gift card. Lastly, severe acute respiratory syndrome coronavirus 2 disease (COVID-19) hindered the ability of patients to receive vaccines during the end of the study period due to stay at home orders and at that time the recommendation from the Centers for Disease Control and Prevention was to not administer non-emergent routine vaccines during the pandemic. ${ }^{7}$

Although limited data was collected on the use of the digital vaccine consent form, this provided limited insight on what can be done in the future to promote this new platform and some ways to improve its usage. The pharmacist and staff are key in educating the public about new services and will need to continue to inform patients about the availability of the digital vaccine consent form.

\section{Conclusion}

The new digital vaccine form was not utilized by most participants. However, further engagement with patients on the availability and use of the digital vaccine consent form may advance digital technologies for pharmacy services.
References

1. Isenor JE, Wagg AC, Bowles SK. Patient experiences with influenza immunizations administered by pharmacists. Hum Vaccin Immunother. 2018; 14(3): 706-711.

2. Isenor JE, Edwards N, Alia T, et al. Impact of pharmacists as immunizers on vaccination rates: a systematic review and meta-analysis. Vaccine. 2016; 34(47): 5708-5723.

3. Spiro S. Digital transformation of pharmacists' clinical services. J Am Pharm Assoc. 2019; 59(2): S8-S12.

4. Lee C, Coughlin JF. Perspective: older adults adoption of technology: an integrated approach to identifying determinants and barriers. J Prod Innov Manage. 2014; 32(5): 747-759.

5. Davies MJ, Collings $M$, Fletcher $W$, et al. Pharmacy Apps: a new frontier on the digital landscape? Pharm Pract. 2014; 12(3): 1-11.

6. Velthoven $\mathrm{MH}$, Cordon C, Challagalla G. Digitization of healthcare organizations: The digital health landscape and information theory. Int J Med Inform. 2019; 124, 49-57.

7. Centers for Disease Control and Prevention. Vaccination Guidance During a Pandemic. https://www.cdc.gov/vaccines/pandemicguidance/index.html Accessed June 9, 2020.

Acknowledgments: None

Funding: None

Conflicts of Interest: None

Treatment of Human Subjects: IRB review/approval required and obtained

Table 1: Demographic Characteristics $(n=36)$

\begin{tabular}{|l|l|}
\hline Characteristic & Participant, $\%$ \\
\hline Gender & \\
\hline Male & $44 \%$ \\
\hline Female & $56 \%$ \\
\hline Age, years & \\
\hline $18-33$ & $11 \%$ \\
\hline $34-49$ & $20 \%$ \\
\hline $50-64$ & $23 \%$ \\
\hline $65+$ & $46 \%$ \\
\hline
\end{tabular}

